\title{
Effect of Hepatitis C Virus Infection in Patients With Cancer: Addressing a Neglected Population
}

\author{
Harrys A. Torres, MDª Parag Mahale, MBBS, MPH ${ }^{\mathrm{a}, \mathrm{b}}$; Boris Blechacz, MDc; Ethan Miller, MD; Ahmed \\ Kaseb, MDd; H. Franklin Herlong, MDc; Nathan Fowler, MDe; Ying Jiang, MSa; Issam I. Raad, MDª and \\ Dimitrios P. Kontoyiannis, MDa
}

\begin{abstract}
Background: Hepatitis C virus (HCV) infection is a neglected disease in patients with cancer. Therefore, this study examined the impact of HCV infections in these patients. Methods: The records of HCV-infected patients with cancer seen at The University of Texas MD Anderson Cancer Center (2008-2011) were reviewed. The outcomes of those who underwent HCV treatment were analyzed. Results: Of 1291 patients who had positive test results for an antibody to HCV (anti-HCV), 744 (58\%) were tested for HCV-RNA; 642 (86\%) of which had chronic HCV infections. Most had solid tumors (72\%) and genotype-1 (G-1) infections (66\%). HCV therapy was administered in 348 patients $(98$ of them after cancer diagnosis). Sustained virologic response (SVR) occurred in 27 (35\%) of the 78 patients treated for whom outcome data were available. Compared with patients who experienced an SVR, more patients who did not were black ( $29 \%$ vs $4 \% ; P=.007)$, had G-1 infections (72\% vs $6 \% ; P<.0001)$, and had higher baseline aspartate aminotransferase (78 vs $47 \mathrm{IU} / \mathrm{L} ; P=.006)$ and alanine aminotransferase levels (71.1 vs $43.3 \mathrm{IU} / \mathrm{L} ; P=.009$ ). Overall, progression to cirrhosis (hazard ratio [HR], 0.38; $P=.03$ ) and portal hypertension (HR, $0.19 ; P=.009)$ was less common in those treated, irrespective of the treatment outcome (SVR or non-SVR). Hepatocellular carcinoma (HCC) developed as a second primary malignancy in $7 \%$ of patients with non-HCC cancer. Conclusions: This is the largest series to analyze HCV infections in patients with cancer. HCV therapy is feasible and prevents liver disease progression in this forgotten population. A treatment algorithm is provided. (J Natl Compr Canc Netw 2015;13:41-50)
\end{abstract}

\section{Background}

Approximately 130 to 170 million persons globally are infected with hepatitis $\mathrm{C}$ virus (HCV). ${ }^{1}$ In the United States, approximately 2.7 to 3.9 million persons (1.0\%$1.5 \%)$ are infected. ${ }^{2}$ Chronic HCV increases the risk for mortality from hepatic and extrahepatic diseases. ${ }^{3}$

The prevalence of HCV infection in patients with cancer ranges from $1.5 \%$ to $32.0 \% .^{4-7}$ Despite the recent interest in $\mathrm{HCV},{ }^{8}$ it is a neglected infection in patients

From the a Department of Infectious Diseases, Infection Control and Employee Health, The University of Texas MD Anderson Cancer Center; ${ }^{\text {b}}$ The University of Texas School of Public Health; and the Departments of 'Gastroenterology, Hepatology and Nutrition, dGastrointestinal Medical Oncology, and eLymphoma/Myeloma, The University of Texas MD Anderson Cancer Center, Houston, Texas.

Submitted March 28, 2014; accepted for publication September 12, 2014. Dr. Torres is a consultant for Gilead Sciences; Merck \& Co., Inc.; Novartis; Astellas Pharma; Pfizer Inc.; Theravance Biopharma, Inc.; Genentech Inc.; and Vertex Pharmaceuticals, and received research grants from Merck \& Co., Inc. and Vertex Pharmaceuticals. Dr. Kontoyiannis is a consultant for Merck \& Co., Inc., a member of a speaker's bureau of Merck \& Co. Inc.; Gilead Sciences; and Pfizer Inc., and received research grants from Merck \& Co., Inc.; Astellas Pharma; and Pfizer Inc. All other authors have disclosed that they have no financial interests, arrangements, affiliations, or commercial interests with the manufacturers of any products discussed in this article or their competitors. with cancer, with little known about its natural history, management, and outcome. Professional societies have published guidelines for the diagnosis, management, and treatment of $\mathrm{HCV}$ infection, ${ }^{9}$ specific sections of which address immunocompromised patients, such as HIV coinfected patients and solid organ transplant recipients, but no recommendations were made for patients with cancer. The FDA-designated HCV-infected special populations include children, HIV-positive pa-

The results of this study were presented in part at the 64th Annual Meeting of the American Association for the Study of Liver Diseases; November 1-5, 2013; Washington, DC.

Author contributions: Dr. Torres designed the study, provided study patients, analyzed and interpreted the data, and wrote the manuscript; Dr. Mahale performed research, analyzed and interpreted the data, performed statistical analysis, designed tables and figures, and wrote the manuscript; Drs. Blechacz, Miller, Herlong, Kaseb, Fowler, Raad, and Kontoyiannis provided patients, analyzed and interpreted the data, and wrote the manuscript; and Dr. Jiang analyzed and interpreted the data, and performed statistical analyses.

Correspondence: Harrys A. Torres, MD, Department of Infectious Diseases, Infection Control and Employee Health, Unit 1460, The University of Texas MD Anderson Cancer Center, 1515 Holcombe Boulevard, Houston, TX 77030. E-mail: htorres@mdanderson.org 
tients, patients with bleeding disorders, liver transplant recipients, and injection drug users, but not patients with cancer.

The most likely reason why no data exist on HCV treatment in patients with cancer is that clinical trials of antiviral or cancer therapy typically exclude these infected patients, partly because their baseline hematologic abnormalities (ie, neutropenia) can be exacerbated by interferon (IFN) alfa and ribavirin-containing $\mathrm{HCV}$ therapy. ${ }^{10}$ Other reasons for excluding these patients is the potential for $\mathrm{HCV}$ to affect the toxicity and/or efficacy of the investigational chemotherapy agents. However, studies have demonstrated the feasibility of using pegylated IFN (pegIFN) alfa plus ribavirin in a subset of patients with cancer, such as hepatocellular carcinoma (HCC) survivors, ${ }^{11}$ or recipients of hematopoietic cell transplants (HCTs). ${ }^{12-14}$ Clinical trials of antiviral treatment exclude $\mathrm{HCV}$-infected patients with cancer, likely because of the impact in long-term outcomes of the underlying malignancy, and limited clinical understanding of potential drug-drug interactions between antivirals and chemotherapy or other immunosuppressive agents in these patients.

Patients with chronic HCV may be at a higher risk of dying from nonliver cancers than the general population. For instance, age-adjusted cancer-related mortality was analyzed for 12,126 chronic HCV-infected patients in the Centers for Disease Control and Prevention's (CDC) Chronic Hepatitis Cohort Study and compared with Multiple Cause-of-Death mortality data for 2006 to 2010 from the National Center for Health Statistics. Of 1496 deaths, 372 (25\%) were from cancer. Compared with the general population, $\mathrm{HCV}$-infected individuals were more likely to die not only from HCC (relative risk [RR], 29.59) but also from nonliver cancers, such as nonHodgkin's lymphoma $(R R, 2.27)$ and rectal $(R R$, $2.60)$, pancreatic $(R R, 1.63)$, and oral cavity or pharyngeal cancers $(\mathrm{RR}, 5.22) .{ }^{15}$

Patients with cancer may benefit from HCVftherapy because persistent transaminase elevation as a result of chronic infection can make cancer treatment complicated. ${ }^{16}$ In addition, successful HCV therapy can cure $\mathrm{HCV}$, prevent its reactivation, ${ }^{17} \mathrm{de}$ lay progression to cirrhosis, ${ }^{12-14}$ and improve overall mortality rates, as observed in the general population of HCV-monoinfected and HIV-HCV-coinfected patients. ${ }^{18,19}$ Few data exist on patients with cancer, but HCV treatment is recommended for all HCT recipients who meet certain criteria, ${ }^{20}$ because HCV infection is associated with a higher risk for nonrelapse-related mortality after allogeneic $\mathrm{HCT}^{21}$

In 2009, The University of Texas MD Anderson Cancer Center established the first US clinic specifically devoted to managing $\mathrm{HCV}$ infections in patients with cancer. ${ }^{22}$ In this study, we determined the treatment outcome of HCV infections in patients with cancer. They hypothesized that HCV infections affect these patients' clinical outcome and that cancer survivors can be safely treated to reduce the risk of liverrelated clinical events.

\section{Methods}

\section{Study Design and Patient Population}

In this retrospective study, the medical records of patients with cancer and HCV infections who were seen at The University of Texas MD Anderson Cancer Center between January 1, 2008, and December 31, 2011, were examined. All patients who had positive test results for an antibody to $\mathrm{HCV}$ (anti-HCV) were identified through a search of the institutional database. Only patients with positive test results for anti-HCV and who had chronic HCV infections (ie, documented HCV-RNA in serum without clinical presentation suggestive of acute infection, or with a history of HCV therapy) were included. The information collected included demographic data, underlying cancer and stage, HCV infection risk factors, cancer therapy, coinfections (eg, hepatitis B virus), clinical presentation, HCV therapy, and outcome. Patients received HCV therapy either at MD Anderson or before referral to the center. Most patients who received HCV therapy at MD Anderson were cancer survivors (ie, persons whose cancer has been in complete remission for $>6$ months since their last cancer treatment and were under surveillance for recurrence). The protocol was approved by the MD Anderson Institutional Review Board.

Patients were tested for anti-HCV using the ABBOTT PRISM HCV assay (Abbott Park, IL). HCVRNA in serum was quantified using a commercially available polymerase chain reaction method (COBAS TaqMan HCV test, version 1; Roche Molecular Systems, Branchburg, NJ). Information on underlying liver disease was sought through available liver biopsy reports. When these reports were not available, CT 
Hepatitis C Infection in Patients With Cancer

scans or liver ultrasound imaging reports were used. All patients with no evidence of cirrhosis at baseline were followed up for evidence of liver disease progression (ie, development of cirrhosis or portal hypertension).

Treatment data were collected until 2011, a predirect-acting antivirals (DAAs) era, reflecting the use of dual combination of pegIFN and ribavirin, or, in a few cases, standard IFN or pegIFN monotherapy. These agents were administered following practice guidelines available at that time for noncancer patients, with the goal of preventing complications and death from HCV infection. ${ }^{9}$

\section{Statistical Analyses}

To determine the predictors of response to $\mathrm{HCV}$ therapy, the characteristics of cancer survivors who attained a sustained virologic response (SVR; ie, absence of HCV RNA in the serum 6 months after discontinuing HCV therapy ${ }^{9}$ ) were compared with those of patients who did not. Categorical variables were compared using the Pearson Chi-square test or Fischer exact test, and continuous variables were compared using an independent 2 -sample Student $t$ test or Wilcoxon rank sum test, as appropriate. Logistic regression was used to determine significant predictors of treatment response, after adjusting for other potential confounders. All variables with a $P$ value less than .2 were included in a multivariable logistic regression model.

The association between HCV therapy and liver disease progression was estimated using Cox regression analysis. The probability of developing cirrhosis or portal hypertension among those who did and did not undergo HCV therapy was estimated using Kaplan-Meier curves; the statistical significance of the difference between the 2 groups was determined using the log-rank test. Unadjusted univariate analyses were conducted, and all variables with a $P$ value less than .2 were included in a multivariable Cox regression model.

All statistical tests were 2-sided and conducted using STATA IC software, version 12.0 (StataCorp LP, College Station, TX). A P value less than .05 was considered statistically significant.

\section{Results}

\section{Study Population}

During the study period, 1291 patients with cancer had positive anti-HCV test results. Of 744 patients tested for HCV-RNA, 642 (86\%) had chronic HCV infections. Most of the 642 patients were men (68\%), non-Hispanic white (65\%), and had genotype 1 (G-1) infections (66\%; Table 1). Solid tumors were the predominant underlying cancer $(n=462 ; 72 \%) ; 26 \%$ of patients had HCC. Of 173 patients (27\%) with hematologic cancer, $61 \%$ had lymphoma. Complete remission of cancer was achieved in 223 patients (35\%). A history of drug abuse was the most common risk factor for $\mathrm{HCV}$ $(60 \%)$ and was more common in men than women (65\% vs $50 \% ; P=.005$; Table 1$)$. Only 35 patients $(5 \%)$ were tested for interleukin-28B polymorphism, with a predominance of the CT genotype noted $(n=17 ; 49 \%)$.

\section{HCV Treatment}

Of 642 patients with HCV infections, 348 (54\%) underwent HCV therapy before $(n=250)$ or after

\begin{tabular}{|c|c|}
\hline Characteristic & Result \\
\hline Male sex, $\mathrm{n}(\%)$ & $435(68)$ \\
\hline Mean age, $y \pm S D$ & $58.3 \pm 9.4$ \\
\hline \multicolumn{2}{|l|}{ Race or ethnicity, n (\%) } \\
\hline Non-Hispanic white & $414(65)$ \\
\hline Black & $110(17)$ \\
\hline Hispanic & $66(10)$ \\
\hline Asian & $30(5)$ \\
\hline Middle Eastern & $15(2)$ \\
\hline Native American & $2(1)$ \\
\hline \multicolumn{2}{|l|}{ Baseline liver biopsy, METAVIR stage, n/total (\%)a } \\
\hline Unknown & $45 / 206(22)$ \\
\hline 0 (no fibrosis) & $14 / 206(7)$ \\
\hline 1 (periportal fibrotic expansion) & $21 / 206(10)$ \\
\hline 2 (periportal septae) & $40 / 206$ (19) \\
\hline 3 (porto-central septae) & $46 / 206(22)$ \\
\hline 4 (cirrhosis) & 40/206 (19) \\
\hline Baseline cirrhosis, $\mathrm{n}(\%)^{\mathrm{a}}$ & $73(17)$ \\
\hline Baseline portal hypertension, $\mathrm{n}(\%)^{\mathrm{a}}$ & $31(8)$ \\
\hline \multicolumn{2}{|l|}{ Cancer type, $\mathrm{n}(\%)$} \\
\hline Hematologic malignancies & $173(27)$ \\
\hline Solid tumors & $462(72)$ \\
\hline Mixed tumors ${ }^{b}$ & $7(1)$ \\
\hline Hematopoietic cell transplant, n/total (\%) & $40(23)$ \\
\hline Mean baseline body mass index $\left(\mathrm{kg} / \mathrm{m}^{2}\right) \pm \mathrm{SD}$ & $27.4 \pm 6.1$ \\
\hline Basal HCV viral load >600,000 IU/mL & $298(69)$ \\
\hline Mean baseline serum cholesterol $(\mathrm{mg} / \mathrm{dL}) \pm S D$ & $167.1 \pm 40.5$ \\
\hline Mean baseline serum triglycerides $(\mathrm{mg} / \mathrm{dL}) \pm \mathrm{SD}$ & $150.5 \pm 98.9$ \\
\hline \multicolumn{2}{|l|}{ Coinfection, $\mathrm{n}(\%)^{\mathrm{a}}$} \\
\hline \multicolumn{2}{|l|}{ Hepatitis B } \\
\hline Exposure & $197(39)$ \\
\hline Infection ${ }^{d}$ & $7(1)$ \\
\hline HIV & $10(2)$ \\
\hline
\end{tabular}

Abbreviation: HCV, hepatitis C virus.

${ }^{\mathrm{a}} \mathrm{For}$ those with data available.

bSolid and hematologic malignancies.

CHepatitis B core antigen positivity.

${ }^{\mathrm{d}}$ Hepatitis $B$ surface antigen positivity.

*Expanded version of Table 1 is available online, with this article, at JNCCN.org. 


\begin{tabular}{|ll|}
\hline \multicolumn{2}{|c|}{ Table 2 Details of HCV Treatment in } \\
Cancer Survivors (N=98) \\
\hline Characteristic & Result \\
\hline HCV treatment regimens, n (\%) & $98(15)$ \\
Monotherapy & $21(21)$ \\
IFN & $21(21)$ \\
Ribavirin & $0(0)$ \\
Combination therapy & $77(79)$ \\
IFN + ribavirina & $76(99)$ \\
IFN + ribavirin + nitazoxanide & $1(1)$ \\
Median treatment duration, wk (range) & $24(2-72)$ \\
Toxicity from HCV treatment, ${ }^{b}$ n/total (\%) & $53 / 98(54)$ \\
Constitutional & $39 / 53(74)$ \\
Hematologic & $39 / 53(74)$ \\
Psychiatric & $21 / 53(40)$ \\
Gastrointestinal & $19 / 53(36)$ \\
Dermatologic & $11 / 53(21)$ \\
Dose reduction of HCV therapy, n/total (\%) & $17 / 32(53)$ \\
Growth factor used, n/total (\%) & $21 / 39(54)$ \\
Erythropoiesis-stimulating agent & $18 / 21(86)$ \\
Granulocyte colony-stimulating factor & $7 / 21(34)$ \\
Thrombopoietic agent & $4 / 21(19)$ \\
Treatment interrupted, ${ }^{\text {c }}$ n/total (\%) & $40 / 61(66)$ \\
Reasons for treatment interruption, n/total (\%) & \\
Hematologic toxicity & $17 / 40(43)$ \\
Constitutional symptoms & $2 / 40(5)$ \\
Depression & $4 / 40(10)$ \\
Chemotherapy initiation & $3 / 40(8)$ \\
Renal insufficiency & $2 / 40(5)$ \\
Othere & $4 / 40(10)$ \\
Unknown side effects of HCV therapy & $8 / 40(20)$ \\
Treatment response known, n/total (\%) & $78(81)$ \\
Sustained virologic response, n/total (\%) & $27(35)$ \\
\hline
\end{tabular}

Abbreviations: HCV, hepatitis C virus; IFN, interferon; pegIFN, pegylated interferon.

ancludes non-pegIFN + ribavirin $(n=19[19 \%])$, pegIFN alfa $2 a+$ ribavirin $(n=49[50 \%])$, and pegIFN alfa $2 b+$ ribavirin $(n=8[9 \%])$. bMore than one type of toxicity occurred in 41 of 53 patients (77\%). cMore than one type of growth factor was administered in 9 of 21 patients $(43 \%)$.

${ }^{\mathrm{d} F o r}$ those with data available.

eOther reasons for treatment interruption include pericardial effusion, hypothyroidism, tumor progression, and elective surgery (1 each).

$(n=98)$ their cancer diagnosis. The details of HCV therapy after cancer diagnosis are depicted in Table 2). Of the cancer survivors who underwent $\mathrm{HCV}$ therapy, most underwent combination therapy with pegIFN alfa $2 \mathrm{a}$ and ribavirin; none received DAAs. HCV therapy was frequently associated with adverse events (54\%), mainly hematologic (74\%) (Table $2)$. Most patients $(77 \%)$ had more than one adverse event. HCV therapy was interrupted in 66\% patients, mostly because of hematologic toxicity $(43 \%)$. Of the 78 patients with known treatment responses, SVR occurred in 27 (35\%; Table 2). The SVR rate was $4 \%$ (1 of 27 patients) for G-1; 59\% (10 of 17) for genotype 2; and $57 \%$ (4 of 7) for genotype
3 infections (Table 3). Patients did not experience SVR due to either treatment interruption because of side effects (24\%) or treatment nonresponse (20\%).

Most treated patients were cancer survivors experiencing complete remission. No cancer survivors experienced a recurrence of the underlying cancer during HCV therapy. No patient experienced cancer progression within 1 year of HCV therapy and only 1 experienced cancer relapse within 2 years after therapy. Selected chemotherapy agents, mainly hormonal, were concomitantly used with HCV treatment (Table 3).

\section{SVR Predictors}

Among patients for whom treatment outcome data were available (Table 3), most of those who did not experience a response to HCV therapy were black ( $29 \%$ vs $4 \% ; P=.007)$ and had more $G-1$ infections (72\% vs $6 \% ; P<.0001$ ), higher baseline aspartate aminotransferase levels (mean, 77.6 vs 46.7 IU/L; $P=.006)$, higher baseline alanine aminotransferase levels (mean, 71.1 vs $43.3 \mathrm{IU} / \mathrm{L} ; P=.009$ ), lower WBC counts (total WBC count $<4000$ cells/mcL; $25 \%$ vs $4 \% ; P=.05)$, and shorter treatment durations (mean, 22.4 vs 27.8 weeks; $P=.04$ ) than those who experienced an SVR. A trend was seen toward treatment failure among men (65\% vs $44 \% ; P=.09$ ) and patients with neutropenia (absolute neutrophil count $<1500$ cells/mcL; $16 \%$ vs 0\%; P=.09). Multivariable logistic regression analysis revealed that those without G-1 infections (odds ratio, 7.2; 95\% CI, 2.2-55.6; $P<.001$ ) were more likely to experience an SVR.

\section{Underlying Liver Disease Progression}

Kaplan-Meier curves were plotted to determine the probability of cirrhosis and portal hypertension among those who were and were not treated (Figures 1 and 2). Patients who were treated for HCV infection were analyzed, irrespective of the time of cancer diagnosis (Figure 1; Table 4). A separate analysis was also conducted by including those who were treated after cancer diagnosis (Figure 2; Table 4).

Overall, unadjusted Cox regression analyses revealed that among those who did not have cirrhosis or portal hypertension at baseline, the hazard of progression to cirrhosis (hazard ratio [HR], 0.31; 95\% CI, $0.18-0.52 ; P<.001$ ) and portal hypertension (HR, 0.26 ; 95\% CI, 0.13-0.5; $P<.001$ ) were lower in the treated group, irrespective of the treatment outcome 


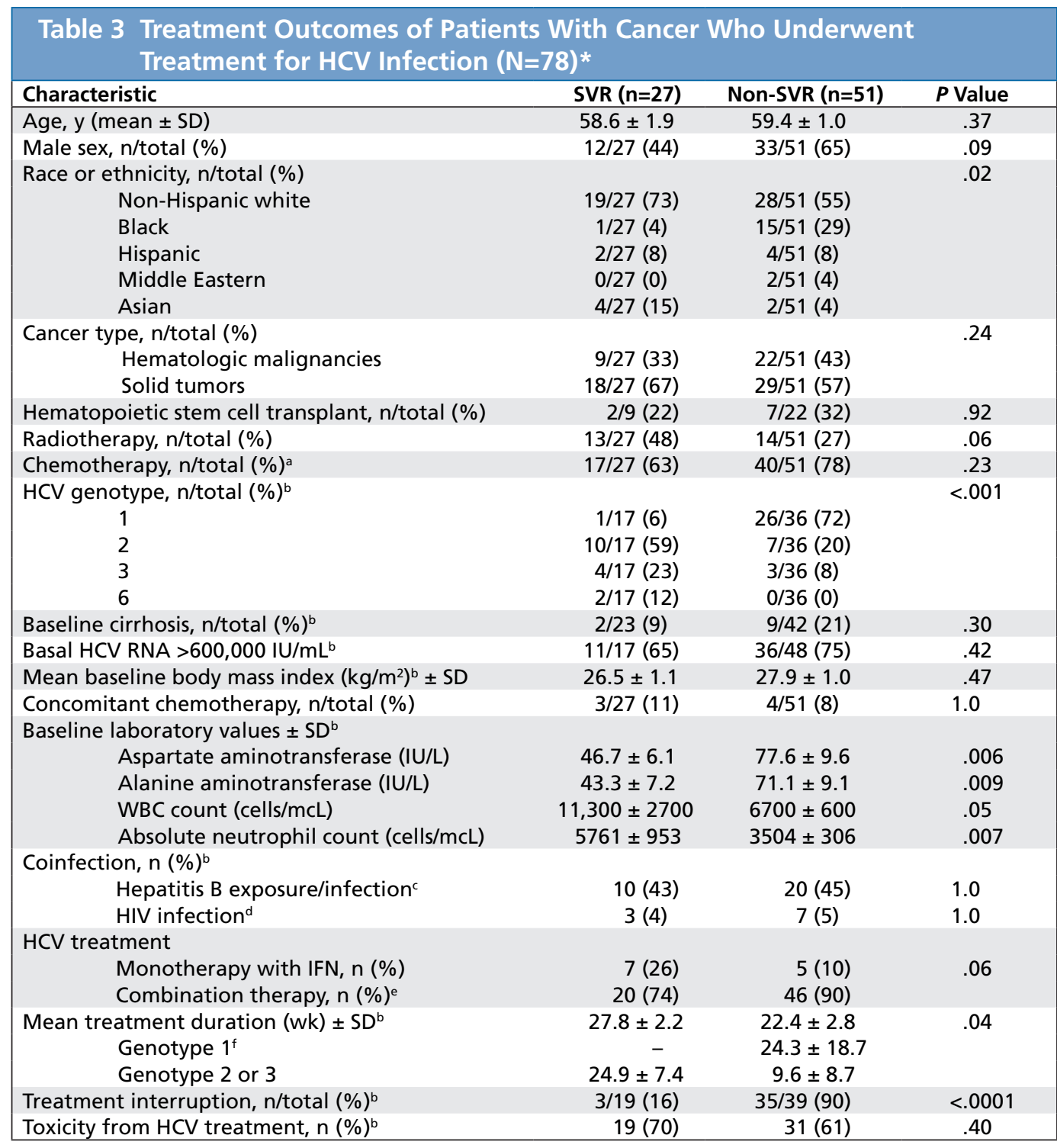

Abbreviations: HCV, hepatitis C virus; IFN, interferon; pegIFN, pegylated interferon; SVR, sustained virologic response.

${ }^{a} A$ patient may have received more than one type of chemotherapeutic agent

${ }^{\mathrm{b}}$ For those with data available.

cHepatitis B core antigen positivity.

dHepatitis B surface antigen positivity.

eIn patients with SVR, includes standard IFN + ribavirin ( $n=3[11 \%])$, pegIFN alfa $2 a+$ ribavirin $(n=15[56 \%])$, and pegIFN alfa $2 b+$ ribavirin $(n=2$ $[7 \%])$. In patients without SVR, includes standard IFN + ribavirin ( $n=13[25 \%])$, pegIFN alfa $2 a+$ ribavirin $(n=27[53 \%])$, pegIFN alfa $2 b+$ ribavirin $(n=5$ $[10 \%])$, and pegIFN alfa $2 a+$ ribavirin + nitazoxanide $(n=1[2 \%])$

The treatment duration was unavailable for the only cancer survivor with a genotype 1 infection who experienced an SVR.

*Expanded version of Table 3 is available online, with this article, at JNCCN.org.

(SVR or no SVR; Table 4). These lower hazards persisted in the multivariable Cox regression model (HR, 0.38; 95\% CI, 0.16-0.93; $P=.03$ vs HR, 0.19; 95\% CI, 0.05-0.66; $P=.009$, respectively; Table 4).

When only those who were treated for HCV infection after cancer diagnosis were included, the hazards of progression to cirrhosis ( $\mathrm{HR}, 0.45 ; 95 \% \mathrm{CI}, 0.22-0.91$; $P<.028)$ and portal hypertension (HR, 0.26; 95\% CI, $0.09-0.75 ; P<.013)$ were lower in the treated group than in the untreated group, irrespective of the treatment outcome (SVR or no SVR; Table 4). These lower hazards persisted in the multivariable Cox regression model 

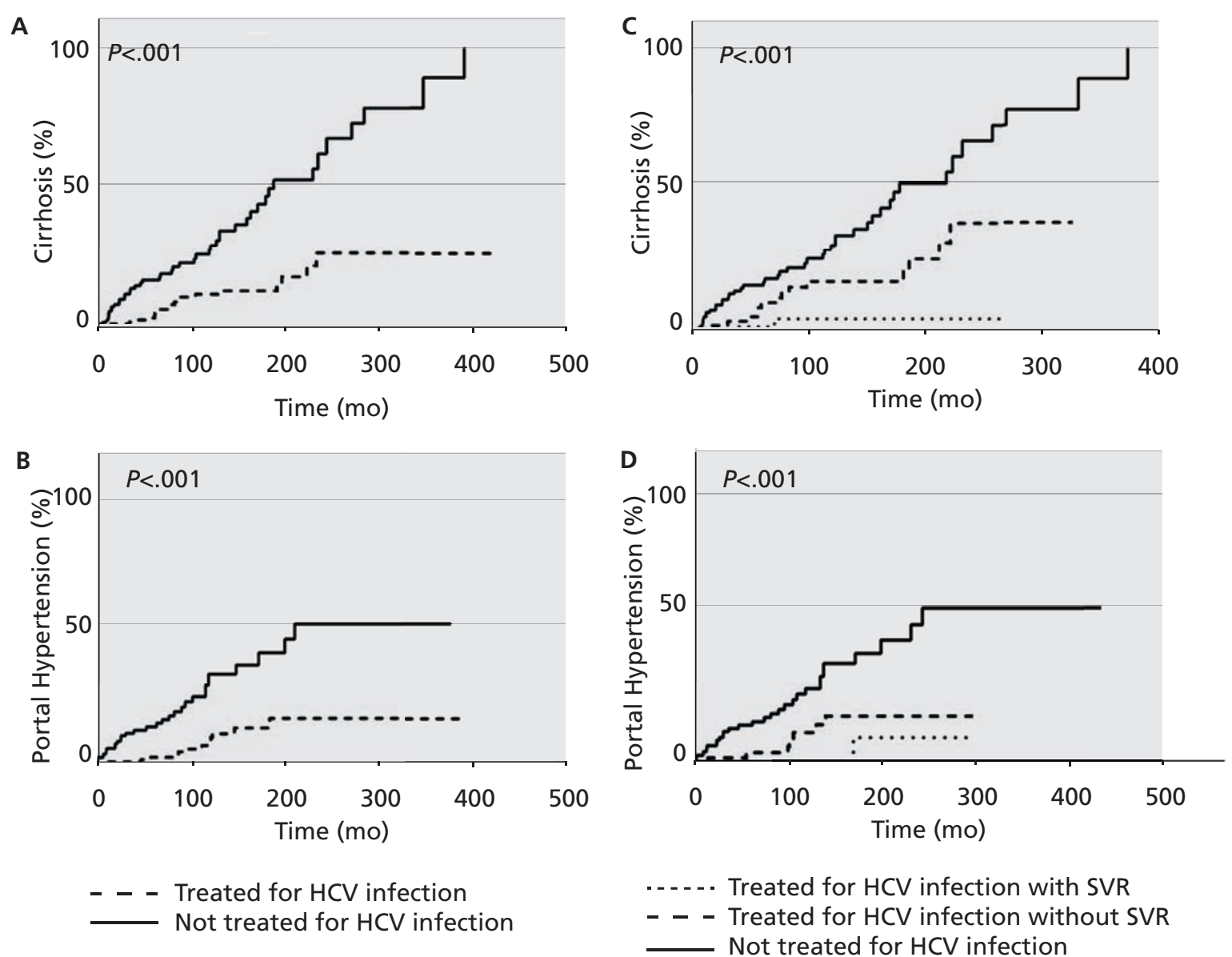

Figure 1 Kaplan-Meier curves for progression to cirrhosis and portal hypertension in hepatitis C virus (HCV)-infected patients with cancer, according to use of HCV therapy. The probability of cirrhosis and portal hypertension increased over time but was significantly higher $(P<.001)$ in patients who did not undergo HCV therapy than in those who did (A and B). Likewise, the probability of underlying liver disease progression differed significantly $(P<.001)$ based on treatment outcome, with those who did not experience a sustained virologic response (SVR) having a higher probability than those who did (C and D).

for progression to portal hypertension (HR, 0.23; 95\% CI, $0.06-0.82 ; P=.02)$ but not cirrhosis (HR, 0.66; $95 \%$ CI, $0.28-1.52 ; P=.33$; Table 4). The final parsimonious regression models are detailed in Supplemental eTables 1 and 2 (available with this article at JNCCN.org). HCC developed as a secondary cancer in 32 of the 476 (7\%) patients with non-HCC cancers.

\section{Discussion}

To our knowledge, this is the largest analysis of the natural history and treatment outcomes of HCV infections in patients with cancer. Infected patients safely underwent HCV therapy after remission. Compared with the general population, SVR was much lower in those with G-1 infections. HCV therapy reduced the risk of liver disease progression in patients with cancer. Developing $\mathrm{HCC}$ as a second primary malignancy is not uncommon among those with baseline non-HCC cancer.

Based on epidemiologic investigations, biological studies, and therapeutic observations, HCV infections seem to be associated with HCC and lymphoma. ${ }^{23-26}$ However, we found that $\mathrm{HCV}$ is not uncommon in patients with other cancers. In a recently reported large community-based cohort study, patients with chronic HCV infections had higher incidences of esophageal, prostate, and thyroid cancers. ${ }^{3}$ The present findings indicate that HCV screening should not be limited to specific groups but should be performed in all patients with cancer.

Overall, a lack of treatment was associated with a higher hazard of progression to cirrhosis or portal hypertension in chronically infected patients, and 
Hepatitis C Infection in Patients With Cancer

\begin{tabular}{|c|c|c|c|c|c|c|c|c|}
\hline \multirow[b]{2}{*}{ Outcome } & \multicolumn{2}{|c|}{ Treated $^{a}$} & \multicolumn{2}{|c|}{ Not Treated } & \multicolumn{2}{|c|}{ Unadjusted } & \multicolumn{2}{|c|}{ Adjusted $^{b}$} \\
\hline & $\begin{array}{l}\text { No. of } \\
\text { Events }\end{array}$ & $\begin{array}{l}\text { Observation } \\
\text { Period } \\
\text { (Person-mo) }\end{array}$ & $\begin{array}{l}\text { No. of } \\
\text { Events }\end{array}$ & $\begin{array}{l}\text { Observation } \\
\text { Period } \\
\text { (Person-mo) }\end{array}$ & HR $(95 \% \mathrm{Cl})$ & $P$ Value & HR $(95 \% \mathrm{Cl})$ & $P$ Value \\
\hline \multicolumn{9}{|c|}{ A. In All Treated Patients $(n=348)$} \\
\hline $\begin{array}{l}\text { Progression to } \\
\text { cirrhosis }^{c}\end{array}$ & 20 & 17,153 & 47 & 12,209 & $0.31(0.18-0.52)$ & $<.001$ & $0.38(0.16-0.93)$ & .03 \\
\hline $\begin{array}{l}\text { Progression } \\
\text { to portal } \\
\text { hypertension }^{\text {d }}\end{array}$ & 12 & 17,579 & 32 & 12,503 & $0.26(0.13-0.50)$ & $<.001$ & $0.19(0.05-0.66)$ & .009 \\
\hline \multicolumn{9}{|c|}{ B. In Patients Treated for HCV Infection After Cancer Diagnosise ( $n=98)$} \\
\hline $\begin{array}{l}\text { Progression to } \\
\text { cirrhosisc }^{c}\end{array}$ & 9 & 5723 & 47 & 12,209 & $0.45(0.22-0.91)$ & .028 & $0.66(0.28-1.52)$ & .33 \\
\hline $\begin{array}{l}\text { Progression } \\
\text { to portal } \\
\text { hypertension }^{\text {d }}\end{array}$ & 4 & 6104 & 30 & 12,375 & $0.26(0.09-0.75)$ & .013 & $0.23(0.06-0.82)$ & .02 \\
\hline
\end{tabular}

Abbreviations: HCV, hepatitis C virus; HR, hazard ratio; SVR, sustained virologic response.

ancluding those who did or did not experience an SVR.

${ }^{b}$ Multivariable Cox proportional hazards regression models adjusted for potential confounders, including age, gender, race, genotype, cancer type, cancer status, baseline laboratory values, coinfections, and baseline METAVIR stage. The proportional hazards assumption was tested with Schoenfeld residuals and through generating an interaction term between the covariate and the log-transformed follow-up time.

'Progression to cirrhosis was assessed in patients who did not have cirrhosis at the time of HCV diagnosis (for section A, $\mathrm{n}=352$; for section $\mathrm{B}$, $\mathrm{n}=268$ ).

dProgression to portal hypertension was assessed in patients who did not have portal hypertension at the time of HCV diagnosis (for section A, $n=371$; for section $B, n=296$ ).

eFinal parsimonious Cox proportional hazards regression models adjusted for potential confounders are described in Supplemental eTables 1 and 2 (available with this article at JNCCN.org).

$\mathrm{HCV}$ therapy tends to prevent this progression, even in those who were treated after cancer diagnosis. This finding is important because liver dysfunction is often associated with abnormal clearance of anticancer agents, and the metabolism of many classes of chemotherapy agents is altered in cirrhosis. ${ }^{27,28} \mathrm{~A}$ previous study in patients without cancer showed a $44 \%$ improvement in liver histology (inflammation and fibrosis) among nonresponders to pegIFN plus ribavirin, ${ }^{29}$ but the benefit on clinical outcomes in these patients is less clear.

Our findings also suggest that HCV-infected cancer survivors should not be excluded from participating in clinical trials of antiviral therapies while under surveillance for cancer recurrence. This is particularly true for patients with G-1 infections because their SVR rate was only $4 \%$ compared with $30 \%$ to $50 \%$ in the general population ${ }^{9}$ and $14 \%$ to $38 \%$ in HIV-coinfected patients treated with pegIFN plus ribavirin. ${ }^{30}$ The SVR rate in the present study was also remarkably lower than the $82 \%$ reported in 11 Taiwanese patients with G-1 infections and nonHCC cancer, ${ }^{11}$ although the different sample sizes in these studies may account for the differences in treatment response. Why G-1-infected patients had such a poor SVR rate is unclear, but certainly these patients may benefit the most from new antiviral agents.

In the present study, treatment data were collected until 2011, the year when DAAs such as the first-generation protease inhibitors (PIs) telaprevir and boceprevir were approved by the FDA..$^{8}$ In our experience, a triple combination-one of these 2 PIs combined with pegIFN and ribavirin - resulted in substantially higher SVR rates but also had a higher incidence of side effects than dual therapy, as reported in patients without cancer. ${ }^{31}$ PegIFN alfa 2 plus ribavirin are still used in cancer survivors with G-1 infection, especially if combined with recently approved DAAs, such as sofosbuvir or simeprevir. ${ }^{32}$ In this population of patients, the use of IFN may be encouraged by some oncologists because of its anticancer activity. ${ }^{33,34}$

We believe that HCV therapy should be offered to patients with cancer in whom this treatment is not contraindicated. Viral eradication may normalize liver function, allowing access to multiple cancer chemotherapies, including agents with hepatic metabolism. ${ }^{35}$ It may also prevent $\mathrm{HCV}$ reactivation, which can occur after chemotherapy and can lead to the discontinuation or dose reduction of potentially 

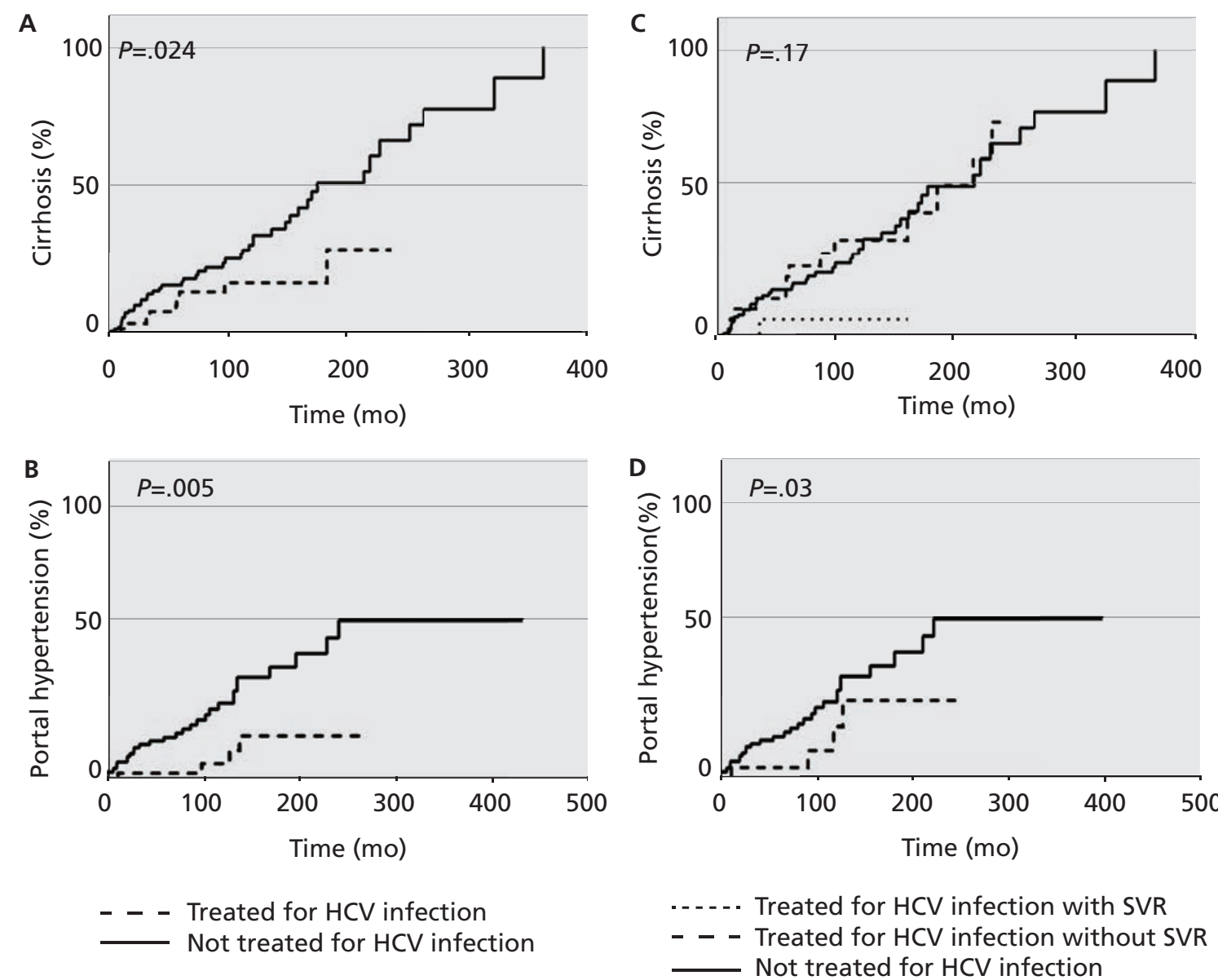

Figure 2 Kaplan-Meier curves for progression to cirrhosis and portal hypertension in hepatitis C virus (HCV)-infected patients with cancer, according to use of HCV therapy after cancer diagnosis. The probability of cirrhosis increased over time and was significantly higher $(P=.024)$ in patients who did not undergo HCV therapy than in those who did (A). However, stratifying based on treatment outcome (sustained virologic response [SVR] vs no SVR) eliminated this statistical significance $(P=.17 ; C)$. The probability of progression to portal hypertension was significantly higher in those who were not treated for HCV infection $(P=.005 ; \mathrm{B})$ even after stratification on SVR status $(P=.03 ; \mathrm{D})$.

life-saving chemotherapy ${ }^{36}$; it may also delay or prevent progression to cirrhosis or hepatic decompensation in patients with cancer, as reported in other patients (eg, normal hosts, solid organ transplant or HCT recipients). ${ }^{9,12-14}$ Furthermore, HCV therapy may reduce the risk of second primary cancers, such as HCC, as described in other infected patients, ${ }^{37}$ and improve the recurrence-free and overall survival rates of patients with selected cancers, such as those with HCV-related HCC. ${ }^{38,39}$

The overall safety and tolerability of $\mathrm{HCV}$ treatment in these patients were similar to those reported for other difficult-to-treat patients (eg, those with HIV coinfection). ${ }^{30}$ Concerns exist about IFN therapy in patients with cancer and HCT recipients, including drug-induced toxicity, cancer relapse, graft compromise, and graft-versus-host disease exacerba- tion. ${ }^{40,41}$ However, IFN-based HCV therapy has been safely used in HCT recipients, including those with underlying hematologic malignancies. ${ }^{12-14}$ In the present study, only one patient experienced cancer relapse within 2 years of HCV therapy.

This study is limited by its retrospective nature, the heterogeneity of the cancer groups analyzed, the lack of treatment protocols, and the small number of patients treated with antivirals after their cancer diagnosis. A referral bias was also possible, wherein only patients likely to survive their cancer were referred for $\mathrm{HCV}$ treatment. Because most of treatment data were collected from available medical records, information on several variables was missing for patients who received treatment outside of MD Anderson. However, using a standardized approach since 2009, we have shown that HCV therapy is feasible in many patients 
Hepatitis C Infection in Patients With Cancer

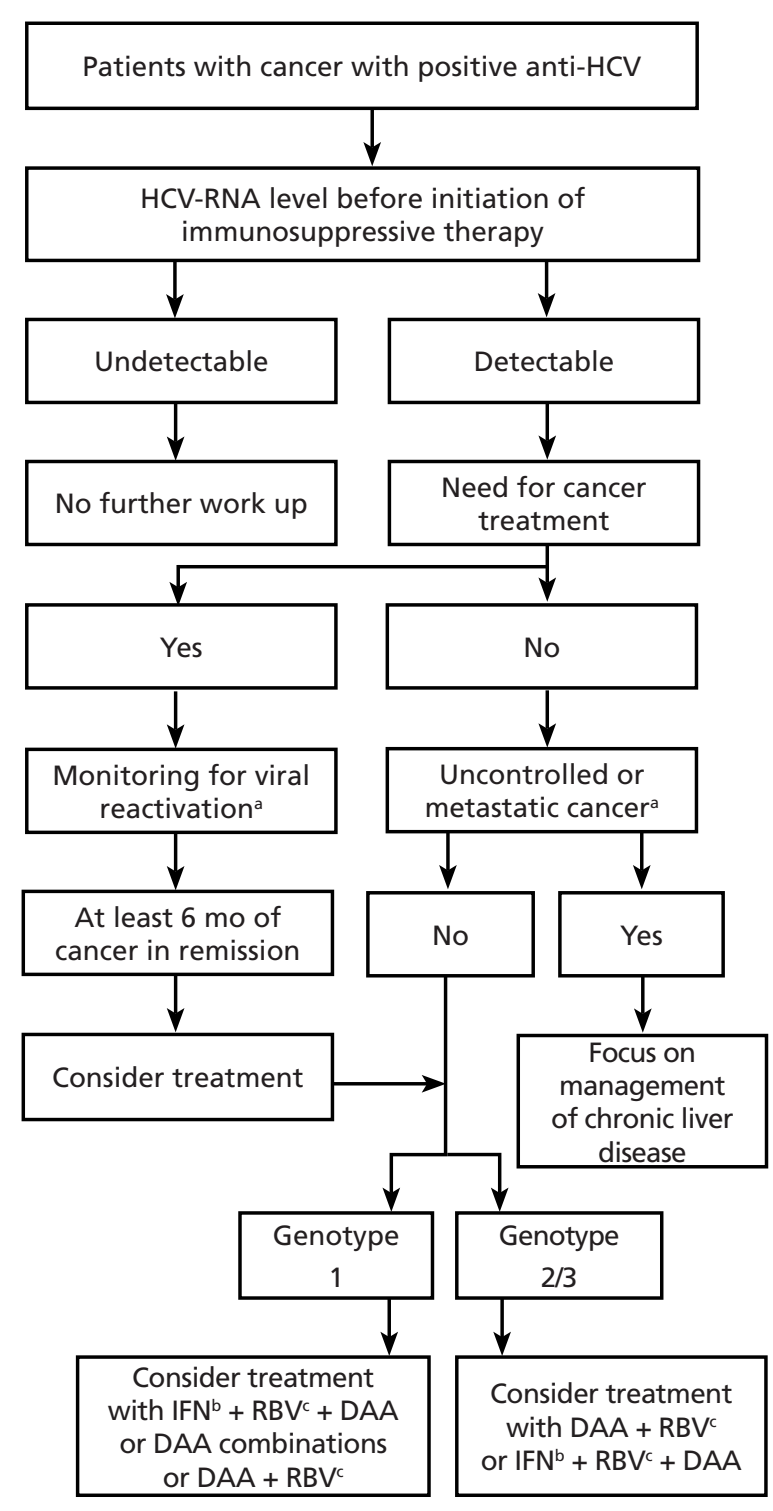

Figure 3 Management algorithm for patients with cancer and chronic HCV infection in 2014. The standard approach is to wait $\geq 6$ months after cancer remission before initiating myelosuppressive HCV treatment with pegylated IFN (pegIFN) and RBV to allow patients' bone marrow to recover. HCV treatment can be administered to HCT recipients who meet the following criteria: complete remission of the original disease, $\geq 2$ y since HCT, no evidence of protracted acute or chronic GVHD, no immunosuppressive therapy for 6 months, and normal blood counts and serum creatinine levels. ${ }^{20}$ The use of pegIFN plus RBV may be encouraged by oncologists because of IFN's anticancer activity ${ }^{33,34}$; however, newer antivirals are urgently needed for patients with genotype 1 infections. The use of DAAs combinations is prudent and recommended in several scenarios (eg, need for IFN-free regimens). ${ }^{32}$

Abbreviations: DAAs, direct-acting antivirals; GVHD, graft-versus-host disease; HCV, hepatitis C virus; HCT, hematopoietic cell transplant; IFN, interferon; RBV, ribavirin.

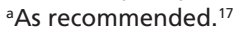

blf not contraindicated. ${ }^{9}$

'As recommended for patients without cancer. ${ }^{9,32}$ with cancer. ${ }^{22}$ The current management algorithm for $\mathrm{HCV}$-infected patients with cancer seen at MD Anderson is depicted in Figure 3.

\section{Conclusions}

This is the largest series to analyze the natural history and outcomes of HCV in patients with cancer. Results showed that HCV therapy is feasible in patients with cancer, and should be administered as recommended. HCV therapy prevents liver disease progression in patients with cancer.

\section{Acknowledgments}

The authors would like to thank Ann Sutton for editorial assistance.

\section{References}

1. Averhoff FM, Glass N, Holtzman D. Global burden of hepatitis C: considerations for healthcare providers in the United States. Clin Infect Dis 2012;55(Suppl 1):S10-15.

2. Smith BD, Morgan RL, Beckett GA, et al. Recommendations for the identification of chronic hepatitis $\mathrm{C}$ virus infection among persons born during 1945-1965. MMWR Recomm Rep 2012;61(RR-4):1-32.

3. Lee MH, Yang HI, Lu SN, et al. Chronic hepatitis $\mathrm{C}$ virus infection increases mortality from hepatic and extrahepatic diseases: a communitybased long-term prospective study. J Infect Dis 2012;206:469-477.

4. Fujii $Y$, Kaku K, Tanaka M, et al. Hepatitis $C$ virus infection in patients with leukemia. Am J Hematol 1994;46:278-282.

5. Markovic S, Drozina G, Vovk M, Fidler-Jenko M. Reactivation of hepatitis $\mathrm{B}$ but not hepatitis $\mathrm{C}$ in patients with malignant lymphoma and immunosuppressive therapy. A prospective study in 305 patients. Hepatogastroenterology 1999;46:2925-2930.

6. Faggioli P, De Paschale M, Tocci A, et al. Acute hepatic toxicity during cyclic chemotherapy in non Hodgkin's lymphoma. Haematologica 1997;82:38-42.

7. Vento S, Cainelli F, Longhi MS. Reactivation of replication of hepatitis $\mathrm{B}$ and $\mathrm{C}$ viruses after immunosuppressive therapy: an unresolved issue. Lancet Oncol 2002;3:333-340.

8. Jensen DM. A new era of hepatitis $C$ therapy begins. N Engl J Med 2011;364:1272-1274.

9. Ghany MG, Strader DB, Thomas DL, Seeff LB. Diagnosis, management, and treatment of hepatitis C: an update. Hepatology 2009;49:1335-1374.

10. Kemmer N, Neff GW. Managing chronic hepatitis $\mathrm{C}$ in the difficult-totreat patient. Liver Int 2007;27:1297-1310.

11. Huang CF, Huang JF, Chen WC, et al. The safety and efficacy of peginterferon plus ribavirin in hepatitis $\mathrm{C}$ patients concomitant with malignancy other than hepatocellular carcinoma: a multicenter study. Hepatol Int 2013;7:180-187.

12. Peffault de Latour R, Levy V, Asselah $T$, et al. Long-term outcome of hepatitis $\mathrm{C}$ infection after bone marrow transplantation. Blood 2004;103:1618-1624.

13. Peffault de Latour R, Asselah T, Levy V, et al. Treatment of chronic hepatitis $\mathrm{C}$ virus in allogeneic bone marrow transplant recipients. Bone Marrow Transplant 2005;36:709-713.

14. Ljungman P, Locasciulli A, de Soria VG, et al. Long-term follow-up of HCV-infected hematopoietic SCT patients and effects of antiviral therapy. Bone Marrow Transplant 2012;47:1217-1221.

15. Allison RD. Increased risk of extra-hepatic cancer mortality among persons with chronic hepatitis $\mathrm{C}$ infection. Presented at: Annual meeting of the American College of Preventive Medicine; February 19-22, 2014; New Orleans, Louisiana. 
Torres et al

16. Locasciulli A, Alberti A. Hepatitis $C$ virus serum markers and liver disease in children with leukemia. Leuk Lymphoma 1995;17:245-249.

17. Torres HA, Davila M. Reactivation of hepatitis B virus and hepatitis C virus in patients with cancer. Nat Rev Clin Oncol 2012;9:156-166.

18. Butt AA, Wang X, Moore CG. Effect of hepatitis $C$ virus and its treatment on survival. Hepatology 2009;50:387-392.

19. Mira JA, Rivero-Juarez A, Lopez-Cortes LF, et al. Benefits from sustained virologic response to pegylated interferon plus ribavirin in HIV/hepatitis $\mathrm{C}$ virus-coinfected patients with compensated cirrhosis. Clin Infect Dis 2013;56:1646-1653.

20. Tomblyn M, Chiller T, Einsele $H$, et al. Guidelines for preventing infectious complications among hematopoietic cell transplantation recipients: a global perspective. Biol Blood Marrow Transplant 2009;15:1143-1238.

21. Ramos CA, Saliba RM, de Padua L, et al. Impact of hepatitis C virus seropositivity on survival after allogeneic hematopoietic stem cell transplantation for hematologic malignancies. Haematologica 2009;94:249-257.

22. Torres HA, Adachi JA, Roach LR, et al. Hepatitis C clinic operated by infectious disease specialists at a comprehensive cancer center: help is on the way. Clin Infect Dis 2012;54:740-742.

23. Davila JA, Morgan RO, Shaib Y, et al. Hepatitis $C$ infection and the increasing incidence of hepatocellular carcinoma: a population-based study. Gastroenterology 2004;127:1372-1380.

24. Gisbert JP, Garcia-Buey L, Pajares JM, Moreno-Otero R. Prevalence of hepatitis $\mathrm{C}$ virus infection in B-cell non-Hodgkin's lymphoma: systematic review and meta-analysis. Gastroenterology 2003;125:1723-1732.

25. Montella M, Crispo A, de Bellis G, et al. HCV and cancer: a case-control study in a high-endemic area. Liver 2001;21:335-341.

26. Antonelli A, Ferri C, Fallahi P, et al. Thyroid cancer in HCV-related chronic hepatitis patients: a case-control study. Thyroid 2007;17:447-451.

27. Donelli MG, Zucchetti M, Munzone E, et al. Pharmacokinetics of anticancer agents in patients with impaired liver function. Eur J Cancer 1998;34:33-46.

28. Lewis JH, Stine JG. Review article: prescribing medications in patients with cirrhosis—a practical guide. Aliment Pharmacol Ther 2013;37:11321156.

29. Manns MP, McHutchison JG, Gordon SC, et al. Peginterferon alfa-2b plus ribavirin compared with interferon alfa-2b plus ribavirin for initial treatment of chronic hepatitis C: a randomised trial. Lancet 2001;358:958965.

30. Matthews GV, Dore GJ. HIV and hepatitis C coinfection. J Gastroenterol Hepatol 2008;23(7 Pt 1):1000-1008.

31. Liang TJ, Ghany MG. Current and future therapies for hepatitis $C$ virus infection. N Engl J Med 2013;368:1907-1917.

32. American Association for the Study of Liver Diseases, Infectious Diseases Society of America. Recommendations for Testing, Managing, and Treating Hepatitis C. Available at: http://hcvguidelines.org/. Accessed March 28, 2014.

33. Ascierto PA, Gogas HJ, Grob JJ, et al. Adjuvant interferon alfa in malignant melanoma: an interdisciplinary and multinational expert review. Crit Rev Oncol Hematol 2013;85:149-161.

34. Talpaz M, Hehlmann R, Quintas-Cardama A, et al. Re-emergence of interferon-alpha in the treatment of chronic myeloid leukemia. Leukemia 2013;27:803-812.

35. van Schaik RH. CYP450 pharmacogenetics for personalizing cancer therapy. Drug Resist Updat 2008;11:77-98.

36. Mahale P, Kontoyiannis DP, Chemaly RF, et al. Acute exacerbation and reactivation of chronic hepatitis $\mathrm{C}$ virus infection in cancer patients. J Hepatol 2012;57:1177-1185.

37. Morgan RL, Baack B, Smith BD, et al. Eradication of hepatitis C virus infection and the development of hepatocellular carcinoma: a metaanalysis of observational studies. Ann Intern Med 2013;158:329-337.

38. Miyatake H, Kobayashi Y, Iwasaki Y, et al. Effect of previous interferon treatment on outcome after curative treatment for hepatitis $\mathrm{C}$ virus-related hepatocellular carcinoma. Dig Dis Sci 2012;57:1092-1101

39. Breitenstein S, Dimitroulis D, Petrowsky H, et al. Systematic review and meta-analysis of interferon after curative treatment of hepatocellular carcinoma in patients with viral hepatitis. Br J Surg 2009;96:975-981.

40. Safdar A. Difficulties with fungal infections in acute myelogenous leukemia patients: immune enhancement strategies. Oncologist 2007;12(Suppl 2):2-6.

41. Klingemann HG, Grigg AP, Wilkie-Boyd K, et al. Treatment with recombinant interferon (alpha-2b) early after bone marrow transplantation in patients at high risk for relapse [corrected]. Blood 1991;78:3306-3311. 